\title{
WhatsApp como ferramenta de ensino: análise do fenômeno da ubiquidade num grupo de estudantes de Pedagogia
}

\begin{abstract}
Débora Valletta ${ }^{1}$
'Programa de Pós-Graduação em Informática na Educação - Universidade Federal do Rio Grande do Sul (UFRGS) - Porto Alegre, RS - Brazil

$\{$ dvalletta@uol.com.br

Abstract. Assuming that the ubiquity context allows the use of personal use tools to insert them with teaching methodologies, the university faces some challenges for initial teacher training. The study investigated the process of instrumentation of the WhatsApp software in disciplines of Degree in Pedagogy to the detriment of the phenomenon of ubiquity. The research is qualitative and exploratory. It was concluded that, among the students, there is evidence that the ubiquity provided by the WhatsApp presents evidence of standardization in the selection of communication tools between the formal and informal learning space and ambience associated with instrumental genesis.
\end{abstract}

Resumo: Assumindo que o contexto da ubiquidade oportuniza o uso de ferramentas de uso pessoal para inseri-las com metodologias de ensino, a universidade se depara com alguns desafios para a formação inicial de professores. $O$ estudo investigou o processo de instrumentalização do artefato WhatsApp com estudantes de Licenciatura em Pedagogia em detrimento do fenômeno da ubiquidade. A pesquisa é qualitativa e de cunho exploratório. Concluiu-se que, entre as estudantes, há evidências que a ubiquidade propiciada pelo WhatsApp apresenta indícios de padronização na seleção de ferramentas de comunicação entre os espaços, formal e informal, de aprendizagem e ambiência associados à gênese instrumental.

\section{Introdução}

Durante a formação inicial (graduação) dos professores, diferentes metodologias de ensino são apresentadas para os estudantes de licenciatura para potencializar novas experiências, por meio das quais se desenvolvem competências e habilidades para o futuro exercício da profissão - ser professor. Nesse processo, as ferramentas utilizadas para fins didáticos permeiam entre a tradição e a inovação.

Reconhecendo-se que o fenômeno da ubiquidade torna difuso o limite entre os espaços formal e informal de aprendizagem, instrumentalizar estudantes de licenciaturas com ferramentas utilizadas para o uso pessoal pode, talvez, ser uma oportunidade para se conectar com esses futuros professores para desenvolver atividades educativas mais significativas para o seu desenvolvimento profissional.

O estudo de Alencar e outros autores (2015) demonstrou que o uso do WhatsApp como ferramenta de ensino potencializa as práticas pedagógicas devido ao nível de interação propiciado pelo aplicativo (App). Kaieski, Grings e Fetter (2015) afirmaram 
VII Congresso Brasileiro de Informática na Educação (CBIE 2018)

Anais do XXIV Workshop de Informática na Escola (WIE 2018)

que o App WhatsApp engajou os estudantes na colaboração e facilitou o sistema de comunicação entre os envolvidos no projeto. Moreno e outros autores (2015) destacam que os aplicativos provocaram uma revolução na forma como os usuários trocam informações, assim como os professores o utilizam como ferramenta em atividades extraclasse e o WhatsApp aproxima estudantes e docentes por ser um artefato de uso pessoal e de fácil instalação nos celulares. Logo, o contexto da ubiquidade oferta oportunidades de exploração, interação e troca de informação em tempo real entre os usuários que fazem o uso desta ferramenta.

Weiser (1991) destacou que a computação ubíqua e pervasiva conectaria o usuário para explorar diferentes trilhas em busca de informação por meio de artefatos capazes de integrar os sistemas de informação e comunicação. Posto o contexto informado por Weiser, então, infere-se que, os estudantes neste estudo utilizaram seus dispositivos pessoais, os smartphones, para realização das atividades do cotidiano acadêmico, ou seja, sem a utilização de computadores. No contexto educacional, o fenômeno da ubiquidade deve ser considerado nos cursos de graduação, pois parte das universidades não está formando professores para atuar na cultura digital (Valletta; Giraffa, 2018). Nesse sentido, percebe-se que, o futuro docente deve atentar-se se as metodologias de ensino que estão articuladas ao contexto social e cultural contemporâneo.

Em relação à Pedagogia, Valletta (2016) e Valletta, Grinkraut e Basso (2017) investigaram o processo de gênese instrumental para compreender os esquemas de uso de ferramentas utilizadas por sujeitos que permeiam o contexto educacional em diferentes áreas do conhecimento. Contudo, alguns estudos revelam que o processo de instrumentalização dos artefatos em usuários que atuam nesse ambiente tem vindo a assumir um caráter cada vez mais próximo do uso de ferramentas de cunho pessoal para o profissional em diferentes áreas do conhecimento e intencionalidades educativas (Spence, 2014; Araújo; Bottentuit Júnior, 2015). A esse cenário relacionam-se questões associadas à formação do futuro docente e à instrumentalização de ferramentas advindas da cultura digital contemporânea como, o aplicativo WhatsApp (artefato).

Nesse âmbito, o presente artigo investigou o processo de instrumentalização do artefato WhatsApp sob a perspectiva de Rabardel (1995) com estudantes de Licenciatura em Pedagogia em detrimento do fenômeno da ubiquidade.

O artigo está organizado em cinco seções: introdução, metodologia, ferramenta como apoio no ensino, algumas considerações e as referências.

\section{Percurso metodológico}

A investigação realizada baseou-se numa metodologia qualitativa e exploratória, utilizando-se o questionário semiestruturado e observação em equipe como técnica de coleta de dados. $\mathrm{O}$ instrumento teve como objetivo investigar os diferentes esquemas de utilização (gênese instrumental) de uma ferramenta categorizada como não educacional numa sequência de atividades com o propósito de compreender como os sujeitos articulam o uso de tecnologia na escola em detrimento da gênese pessoal. De acordo com Marconi e Lakatos (2010) esse tipo de observação favorece a eficácia de sua realização e o registro sob diferentes ângulos, o que oportuniza o confronto dos dados na análise. 
VII Congresso Brasileiro de Informática na Educação (CBIE 2018)

Anais do XXIV Workshop de Informática na Escola (WIE 2018)

Participaram da sequência de atividades seis estudantes de Pedagogia que cursaram a disciplina Tecnologias da Informação e Comunicação em Educação I (TIC I) no primeiro semestre de 2018. A idade dos estudantes compreende entre 22 e 50 anos. Destes, quatro tem experiência na docência como auxiliar de ensino e dois nunca tiveram contato com escolas.

O experimento foi realizado entre março e maio de 2018. O planejamento da disciplina foi alinhado com/entre os estudantes da seguinte forma: apresentação da dinâmica das aulas teóricas e práticas; disponibilização dos espaços virtuais' formais da instituição de ensino e ferramenta de apoio/comunicação à escolha do grupo - o aplicativo WhatsApp; metodologia de ensino híbrido e as lições/tema de casa.

A sequência de atividades nas aulas presenciais aconteceu da seguinte forma:

- As aulas foram organizadas por datas (conforme o calendário escolar) com o apoio da ferramenta Google Sala de Aula;

- Sequência de atividades teóricas: O arquivo ou hiperlink contendo as leituras básicas e/ou complementares da disciplina TIC I referente as discussões da semana foram disponibilizadas nos tópicos/datas previamente (semestre) para que os estudantes pudessem ler em casa como lição, ou seja, antes da aula presencial;

- Sequência de atividades práticas: Os hiperlink da ferramenta para provocar as discussões, estudos ou experimentação foram inseridas de forma gradativa (aula a aula - dia da aula presencial);

- Aula dialogada a partir das experiências pessoais e profissionais dos estudantes;

- Preenchimento do diário de bordo ao final de cada aula;

- Tarefas/temas para casa (via Google Sala de Aula) para os estudantes rever as atividades que foram desenvolvidas na sala de aula contendo desafios para aplicação em diferentes contextos;

- Diálogo sobre o envio e recebimento de mensagens instantâneas por meio do aplicativo WhatsApp ao longo da semana. O grupo foi criado por um dos estudantes para tirar dúvidas sobre as atividades (sala de aula ou fora dela). O professor realizou a intervenção pedagógica somente quando o grupo demorava a responder ao colega ou para mediar conflitos entre eles.

- O restante do tempo foi utilizado para a organização do espaço de aprendizagem e a aplicação do questionário semiestruturado.

Os dados foram coletados a partir do questionário semiestruturado disponibilizado para os sujeitos contendo questões fechadas e abertas para que eles pudessem responder sem a presença do entrevistador, como sugerem Marconi e Lakatos (2010). Os dados foram analisados após 14 semanas de aulas sob a perspectiva da análise textual discursiva de Moraes e Galiazzi (2007). A análise possibilita a

\footnotetext{
'Espaços virtuais neste trabalho são entendidos como ambientes em que os usuários acessam, comunicam, interagem e trocam informações ou conhecimento por meio de ferramentas de conexão, tais como: internet, redes sociais, bluetooth, inteligência artificial, Near Field Communication (NFC), jogos online, plataformas entre outros.

${ }^{2} \mathrm{O}$ aplicativo Google Sala de Aula possibilita aos educadores criar turmas, postar tarefas, avisos e feedback de forma fácil e dinâmica.
} 
VII Congresso Brasileiro de Informática na Educação (CBIE 2018)

Anais do XXIV Workshop de Informática na Escola (WIE 2018)

construção e desconstrução de metatextos originando em categorias a priori e emergentes. O diário de bordo foi (re) lido para articular com os dados do questionário.

Por fim, os resultados foram discutidos a partir das tessituras da decodificação de cada unidade e a observação da manipulação entre os artefatos ao longo da sequência de atividades.

\section{WhatsApp como ferramenta de ensino}

O artefato denominado de WhatsApp é um aplicativo composto por um conjunto de ferramentas (dentro do Apps) que possibilita o usuário a interagir e trocar informações em diferentes formatos (texto, imagem e vídeo) entre grupos e/ou individualmente.

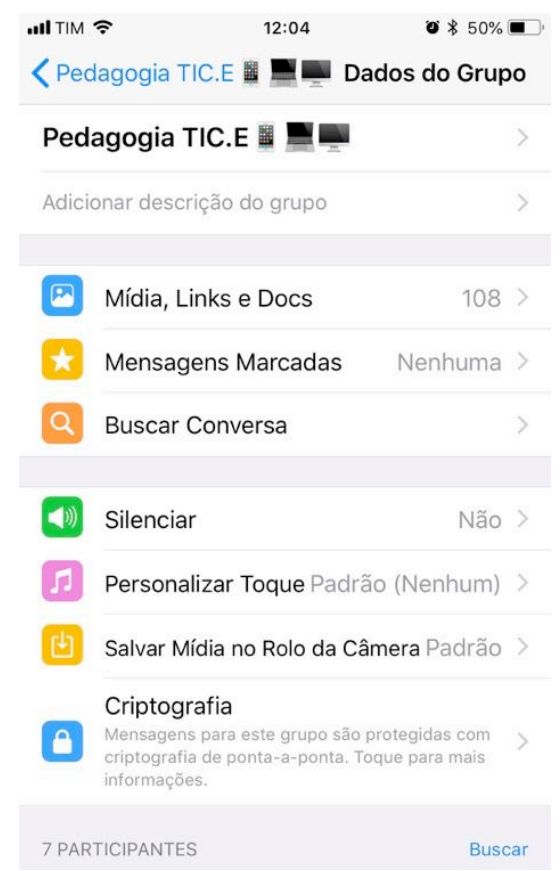

Figura 1 - Grupo criado por uma estudante de Pedagogia

O aplicativo pode ser baixado em qualquer dispositivo móvel cujas especificações possibilita acessar o Apps da loja online AppleStore e GooglePlay. Há diferenças entre as possibilidades de uso no sistema operacional iOS e Android, porém, nada que impeça os estudantes na trocar mensagens em diferentes tipos de linguagens multimodais. O WhatsApp é classificado pelas lojas online como redes sociais. Embora o aplicativo seja categorizado como uma ferramenta de comunicação, o App foi sugerido por um dos estudantes e usado pelo professor como ferramenta de ensino para apoiar no processo de aprendizagem deles [estudantes] - para tirar dúvidas das atividades. Por outro lado, entende-se que há outras ferramentas formais de ensino como os ambientes virtuais de aprendizagem (AVA) que possibilitam a troca de informações entre os usuários como o Moodle, porém, a melhor ferramenta de comunicação sugerida ${ }^{3}$ por este grupo de estudantes (sujeitos) foi o WhatsApp. Nesse sentido, destaca-se a importância de compreender a instrumentalização destes sujeitos que transitam entre a gênese pessoal e profissional para, talvez, se avançar na formação docente em relação ao uso desse tipo de tecnologia aplicada à educação.

${ }^{3}$ Segundo os dados analíticos no site Apptopia (2018) o WhatsApp é considerado o aplicativo que os usuários permanecem mais tempo utilizando a ferramenta no mundo. 
VII Congresso Brasileiro de Informática na Educação (CBIE 2018)

Anais do XXIV Workshop de Informática na Escola (WIE 2018)

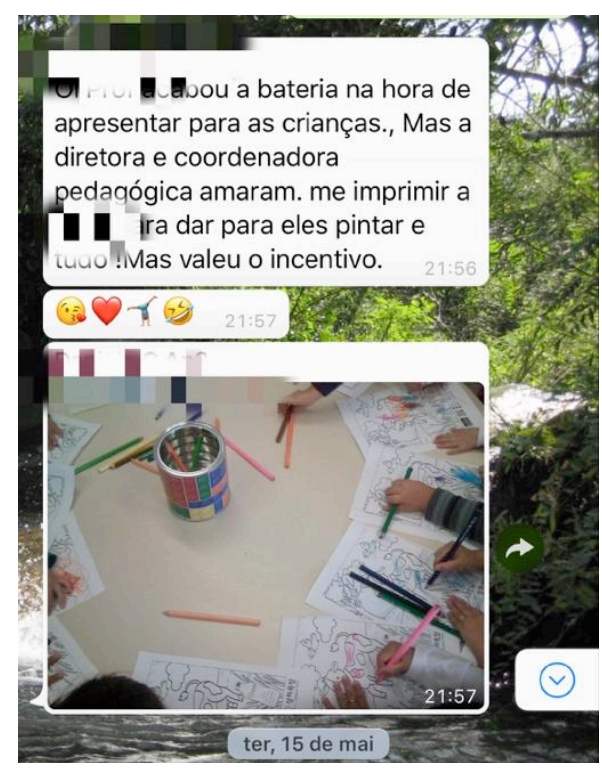

Figura 2 - Fragmento da interação entre os sujeitos

A interface simples e intuitiva do aplicativo possibilita enviar e trocar informações de forma dinâmica e atraente em tempo real. A Figura 2 apresenta uma das atividades compartilhada com/entre os colegas e professor. Trata-se de uma experiência realizada em sala de aula utilizando um aplicativo de realidade aumentada para crianças entre quatro e cinco anos que foi recriada pelo sujeito S2 e implementada ${ }^{4}$ na escola em que realizou a disciplina Estágio Supervisionado na Educação Infantil I, em menos de duas semanas. Nesse sentido, destaca-se que o contexto da ubiquidade a oportunizou [estudante, S2] compartilhar suas produções entre os colegas da sua turma.

No decorrer da sequência de atividades, os estudantes, puderam dividir informações e conhecimentos com o colega que havia faltado à aula.

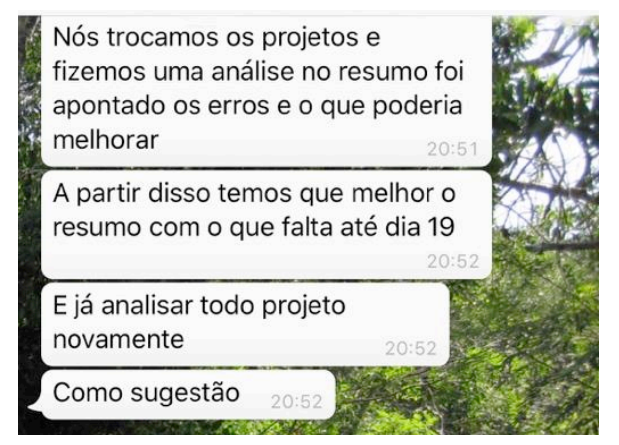

Figura 3 - Fragmento da troca entre pares

$\mathrm{Na}$ análise do conteúdo trocado entre o grupo, referente às habilidades que os sujeitos possuem sobre a tecnologia para uso pessoal, foi identificado como aspecto importante, percebeu-se que a estratégia pedagógica pode influenciar, talvez, na decisão do professor em relação à alteração de atividades práticas para que possa se conduzir de fato a uma aula dialogada.

\footnotetext{
${ }^{4} \mathrm{Na}$ ocasião a estudante apresentou a ferramenta para a diretora da escola e realizou uma atividade experimental com as crianças. Fato que motivou a gestão investir na inovação - uma prática mais contemporânea e diferente de contar histórias.
} 
VII Congresso Brasileiro de Informática na Educação (CBIE 2018)

Anais do XXIV Workshop de Informática na Escola (WIE 2018)

A ideia de que todos os sujeitos sabiam usar a ferramenta escolhida por eles para o uso pessoal (WhatsApp) foi equivocada e, no processo de ensino houve a necessidade de retomar questões associadas à técnica e aos esquemas mentais de uso de cada sujeito.

A Figura 4 apresenta um fragmento das palavras mais frequentes trocadas no espaço digital e que estão relacionadas com as dúvidas sobre o entendimento de uso de outras ferramentas propostas no Apps Google Sala de Aula. O uso do grupo no WhatsApp o ajudou no processo de instrumentalização de outras ferramentas fenômeno advindo da ubiquidade.

Na mediação notou-se que os receios pessoais de uso da ferramenta no decorrer do período de experiência foram desvelados. Nesse sentido, Rabardel (1995) enfatiza que cada sujeito constrói a forma (conexões mentais) como utilizará o artefato, processo denominado como gênese instrumental.

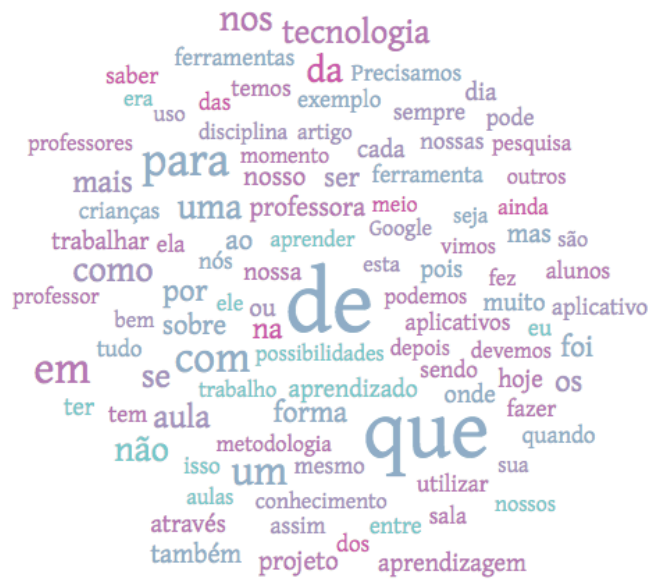

Figura 4 - Palavras mais frequentes trocadas por meio do WhatsApp

Foi possível observar que quatro sujeitos têm pouca familiaridade com a tecnologia para o uso pessoal e as tarefas para serem executadas fora da sala de aula tornaram-se o maior desafio, uma vez que, em alguns momentos, os estudantes necessitavam estabelecer diferentes operações mentais entre a manipulação dos artefatos e o conteúdo a ser aprendido na sala de aula.

No que se refere ao uso pessoal do WhatsApp os sujeitos destacaram (os relatos estão na íntegra):

- S1 - Não tenho muito desafios, a minha dificuldade maior é encontrar aplicativos que eu precise;

- $S 2$ - inventar;

- S3 - meu celular não permitir que eu possa baixar aplicativos que eu gostaria e não buscar outras alternativas;

- S4 - Não tenho facilidade, ao mesmo tempo em que sei que ela é importante ela é um desafio que tem que ser explorada para compreender;

- S5 - Descobrir as ferramentas ocultas existentes no Google que são excelentes para o aprendizado;

- S6 - facilidade de acesso e desafios de entendimento.

É pertinente destacar que alguns padrões de uso foram identificados, tais como: as características pessoais dos sujeitos, surgindo várias vezes expressões como 
VII Congresso Brasileiro de Informática na Educação (CBIE 2018)

Anais do XXIV Workshop de Informática na Escola (WIE 2018)

"experimental", "sei o básico" ou "meu celular é antigo...", ilustrando a ideia de que, por vezes, o processo de instrumentalização para o uso pessoal é, sobretudo, tomado em consideração ao que o sujeito pensa ou quer, e não tanto em relação ao que a ferramenta é capaz de propiciar no ensino ou aprendizagem.

Os dados analisados e as falas apresentadas pelos sujeitos 1 e 3 indicaram evidências de que o artefato para o uso pessoal, talvez, possibilite parte da transposição para o uso acadêmico/profissional. A pesquisa revelou que cinco dos sujeitos acessam as informações (pessoal, profissional ou acadêmico) por meio do dispositivo pessoal. Logo, infere-se que este comportamento está associado ao fenômeno da ubiquidade.

Nesse sentido, os resultados obtidos corroboram com o destaque de Rabardel (1995) em relação ao domínio da tecnologia estar associado aos esquemas mentais que o sujeito faz do seu uso. O autor informa que o sujeito é instrumentalizado na medida em que o artefato é utilizado em sua prática com os estudantes. Fato ocorrido com os sujeitos S2 (Figura 2) e S6.

Ao longo da sequência de atividades obteve-se 1022 mensagens enviadas e/ou recebidas entre o grupo. Sendo que: S1 (n. 290); S2 (n. 270); S3 (n. 90); S4 (n. 46); S5 (n. 36); S6 (n.31) e o professor (n. 259). Notou-se que o sujeito S1 (n. 290) é o que mais tem domínio da ferramenta WhatsApp, bem como apresenta facilidade de uso em outras utilizadas na sala de aula ou aquelas que estão associadas à tarefa/tema de casa. Por outro lado, o sujeito $\mathrm{S} 2$ (n. 270) tem mais dificuldade em usar a maioria das ferramentas utilizadas ao longo do curso, porém, a interação no grupo criou oportunidades para que o mesmo continuasse engajado e conseguisse concluir as atividades dentro e fora da sala de aula.

No início das atividades o WhatsApp era apenas um artefato para o sujeito S2 para comunicar-se com os colegas e professor. Foi na $11^{\mathrm{a}}$ aula que o $\mathrm{S} 2$ começou a compartilhar o conhecimento desenvolvido na sala de aula utilizando o WhatsApp como meio facilitador de seu percurso de aprendizagem junto as ferramentas.

Em relação ao uso de tecnologias em geral para o uso pessoal, os sujeitos consideram-se como usuários que desenvolveram diferentes níveis de habilidades abaixo do básico; adequado e avançado - em enviar uma mensagem via WhatsApp, tirar foto do smartphone, entre outros. Sendo que quatro atingiram o nível adequado, um ficou abaixo do básico e outro avançado. O sujeito cujo nível é abaixo do básico é aquele que havia demonstrado maior dificuldade em lidar com a ferramenta, enquanto o que se encontra no nível avançado é o mesmo que indicou ter maior facilidade.

Os estudantes, em diferentes níveis de aprendizagem, foram instrumentalizados de acordo com o seu ritmo devido ao fenômeno da ubiquidade. Com apoio do professor e principalmente dos colegas puderam compartilhar suas angústias e conquistas no percurso da sequência de atividades - dentro e fora da sala de aula.

A partir das tessituras entre a metodologia de ensino e a ferramenta sugerida pelo estudante, pode-se compreender como os futuros docentes realizaram a sequência de atividades com o apoio da tecnologia. Papert (2008, p. 67) salienta que, sob a perspectiva dos professores, o desafio de entender essa interação (professor e máquina) "favorece a mudança e aqueles que resistem a ela". Nesse sentido, o fenômeno da ubiquidade, talvez, esteja contribuindo para a mudança do método de ensino tradicional. 
VII Congresso Brasileiro de Informática na Educação (CBIE 2018)

Anais do XXIV Workshop de Informática na Escola (WIE 2018)

\section{Considerações finais}

$\mathrm{Na}$ sequência de atividades deste estudo, relacionada à influência da ubiquidade e ao tipo de experiência que o usuário tem com a ferramenta de uso pessoal, verificou-se com base na análise dos dados que, gostos, medos e valores culturais caracterizam o futuro professor e, talvez, poderão ser identificados como um importante processo na instrumentalização do artefato "do momento" para a formação docente.

Cogita-se que, pelos apontamentos feitos neste artigo, um dos conceitos importantes para discutir o fenômeno da ubiquidade é a experiência prática do futuro pedagogo. Uma vez que foi possível observar que ao aplicar a sequência de atividades com os estudantes, as tarefas eram concluídas de forma gradativa e que foi preciso propiciar situações de aprendizagem para o desenvolvimento de habilidades socioemocionais que favorecessem os sujeitos a prosseguirem na realização das atividades teóricas e práticas, principalmente para com aqueles que demonstraram dificuldades técnicas ou comportamentais. A interação do grupo no WhatsApp foi fundamental para aproximar a teoria e a prática proposta na sequência de atividades em diferentes tempos e espaços (dentro e fora da sala de aula).

É possível afirmar, portanto, que o fenômeno da ubiquidade colocou em cena a Pedagogia em meio a uma das ferramentas de comunicação instantânea, o aplicativo WhatsApp, apresentando indícios de que elas [ferramentas] provocam o pensar e padronizam a comunicação entre os espaços, formal e informal, de aprendizagem. Destaca-se que a possibilidade de constante troca de informações, em qualquer dia e horário da semana, apresenta evidências de aspectos, também, associados a gênese instrumental - da pessoal à profissional. A intenção deste artigo foi, pois, aproximar o leitor do fenômeno da ubiquidade provocada pela computação ubíqua e pervasiva via dispositivos móveis e seus aplicativos como, o WhatsApp.

\section{Referências}

Alencar, G. et al. (2015). WhatsApp como ferramenta de apoio ao ensino. In: CONGRESSO BRASILEIRO DE INFORMÁTICA NA EDUCAÇÃO (CBIE), 4, 2015, Maceió. Anais...Maceió: Centro Cultural e de Exposições Ruth Cardoso, 2015. p. 787-795.

Araújo, P. C.; Bottentuit Júnior, J. B. (2015). O aplicativo de comunicação Whatsapp como estratégia no ensino de Filosofia. Temática, Universidade Federal da Paraíba, Ano XI, n. 2, p. 11-23, 2015.

Moreno, F. et al. (2015). Tical: Chatbot sobre o atlas linguístico do Brasil no whatsapp. (2015). In: BRAZILIAN SYMPOSIUM ON COMPUTERS IN EDUCATION (Simpósio Brasileiro de Informática na Educação-SBIE), 26, 2015, Maceió. Anais...Maceió: Centro Cultural e de Exposições Ruth Cardoso, 2015. p. 279-288.

Kaieski, N; Grings, J. A.; Fetter, S. A. (2015). Um estudo sobre as possibilidades pedagógicas de utilização do WhatsApp. RENOTE - Novas Tecnologias na Educação, Universidade Federal do Rio Grande do Sul, v. 13, n. 2., p. 1-10, Dez. 2015.

Marconi, M. A.; Lakatos, E. M. (2010). Fundamentos de metodologia científica. 7 ed. São Paulo: Atlas, 2010. 320p. 
VII Congresso Brasileiro de Informática na Educação (CBIE 2018)

Anais do XXIV Workshop de Informática na Escola (WIE 2018)

Moraes, R.; Galiazzi, M. C. (2007). Análise textual discursiva. Ijuí: Editora Unijuí, 2007. 264p.

Papert, S. (2008). A Máquina das Crianças: repensando a escola na era da informática. Tradução Sandra Costa. Porto Alegre: Artmed, 2008. 216p.

Rabardel, P. (1995). Les hommes et les technologies; approche cognitive des instruments contemporains. Paris: Armand Colin, 1995. 239p.

Spence, N. C. F. M. (2014). O WhatsApp Messenger como recurso no ensino superior: narrativa de uma experiência interdisciplinar. Revista de Educação do Vale do Arinos, Universidade do Estado do Mato Grosso, v. 1, n. 1, p. 3-14, 2014.

Valletta, D.; Giraffa, L. (2018). O contexto da ubiquidade chega à escola: reflexões para a formação e prática docente. Curitiba: Editora Appris, 2018. 109p.

(2016). Aplicativos para Tablets: ferramentas para o pensar. RENOTE - Novas Tecnologias na Educação, Universidade Federal do Rio Grande do Sul, v. 14, n. 2, p. $1-10,2016$.

.; Grinkraut, M.; Basso, M. (2017). A lógica de programação como ferramenta para o pensar: entre o concreto e o virtual. In: WORKSHOP DE INFORMÁTICA NA ESCOLA, 23, 2017, Recife. Anais...Recife: UFPE., 2017. p. 785-794.

Weiser, M. (1991). The Computer for the 21st Century. Scientific American Ubicomp, v. 265, n. 3 , p. 66-75, 1991. 